\title{
CORRECTION
}

\section{Correction to: No added value using SPECT/CT to analyze persistent symptoms after anterior cruciate ligament reconstruction}

\author{
Christian Egloff $^{1} \cdot$ Lukas Huber $^{2} \cdot$ Markus Wurm $^{3} \cdot$ Geert Pagenstert $^{4,5,6}$
}

Published online: 20 March 2019

○) Springer-Verlag GmbH Germany, part of Springer Nature 2019

\section{Correction to: \\ Archives of Orthopaedic and Trauma Surgery https://doi.org/10.1007/s00402-019-03117-7}

The shared first authorship of Christian Egloff, MD and Lukas Huber was always planned and correctly acknowledged in the manuscript submitted to AOTS. However, it has been inadvertently forgotten to declare the shared first authorship correctly at the author's page by the corresponding author during manuscript revisions.

Christian Egloff and Lukas Huber shared first authorship.

The original article can be found online at https://doi.org/10.1007/ s00402-019-03117-7.

Christian Egloff

Christian.egloff@usb.ch

Lukas Huber

Lukas.huber@unibas.ch

Geert Pagenstert

geert.pagenstert@unibas.ch

1 Department of Orthopedics and Traumatology, University Hospital Basel, Spitalstrasse 214031 Basel, Switzerland

2 University of Basel, Basel, Switzerland

3 Department of Orthopaedic Sports Medicine, Technische Universität München (TUM), Ismaninger Str. 22, 81675 Munich, Germany

4 Department of Clinical Research, University of Basel, Schanzenstr. 55, 4056 Basel, Switzerland

5 Clarahof Clinic of Orthopaedic Surgery,

Merian-Iselin-Hospital Swiss Olympic Medical Center, Foehrenstr. 2, 4054 Basel, Switzerland

6 Knee Institute Basel, Mittlere Str. 129, 4056 Basel, Switzerland 